\title{
Herceptin: HERalding a new era in breast cancer care but at what cost?
}

\author{
Lisa Hutchinson and Vincent T DeVita, Jr
}

This year's ASCO meeting saw the presentation of data among the most celebrated in the field of oncology, which received rapturous applause and a standing ovation. In short, the now published data of the randomized HERA trial, and the joint B-31 and N9831 trials showed remarkable results-a staggering $46 \%$ reduction in breast cancer recurrence and $33 \%$ reduction in risk of death was seen in women receiving trastuzumab (Herceptin ${ }^{\circledR}$, Genentech Inc., San Francisco, CA) plus adjuvant therapy after surgical treatment of primary breast cancer-even after a relatively short follow-up of 1-3 years. ${ }^{1,2}$ So impressive were these data that the safety monitoring committees stopped the trials after the first interim analysis and all patients in the control arm were offered trastuzumab. One ASCO presenter commented "As a result of these trials many more women will receive Herceptin ${ }^{\circledR}$ ". But will they? In spite of these results and the reassuring proclamations that this drug will be made available on the NHS, ${ }^{3}$ several reports in the UK press reveal that women are being denied access to the drug.

Annually, around 40,000 women in the UK are diagnosed with breast cancer and if trastuzumab was introduced immediately, this treatment would save around 1,000 lives. ${ }^{3,4}$ There are, of course, several regulatory hurdles, which will dictate how quickly trastuzumab will become available. Primary Care Trusts in the NHS will be partly responsible for deciding whether they will fund this treatment. Treatment does not come cheap; it is estimated to cost around £21,800 per patient per year, ${ }^{5}$ a financial burden for any country, especially for one with a government-funded healthcare system. The UK government has promised that all women diagnosed with earlystage breast cancer will be tested for HER-2/neu protein overexpression, partly as a consequence of the decision by the Somerset Coast Primary Care Trust to allow a patient with early-stage breast cancer to receive the drug. ${ }^{6}$

At the moment, the National Institute for Clinical Excellence has authorized trastuzumab
It seems a shame to allow trastuzumab use only in a situation in which it can prolong life but not when it could save thousands of lives

L Hutchinson is Editor and VT DeVita, Jr is Editor-in-Chief of Nature Clinical Practice Oncology.

Competing interests

The authors declared they have no competing interests.

www.nature.com/clinicalpractice doi:10.1038/ncponc0378 use only in women with advanced metastatic disease, where treatment is not curative. It seems a shame to allow trastuzumab use only in a situation in which it can prolong life but not when it could save thousands of lives. In light of the impressive trial data, thousands more lives could be saved if women with early-stage cancer received trastuzumab.

Unfortunately, cancer patients not receiving adequate access to adjuvant therapy, or not being encouraged to use it, is not new in the UK. Some years ago, a report from the UK noted that, stage for stage, mortality rates for breast cancer were higher in the UK than in the US; this is partly attributed to a dearth of trained medical oncologists to apply adjuvant treatment. ${ }^{7}$ The fact that the use of adjuvant therapy for stage II breast cancer did not occur in the UK until data were seen on many thousands of patients contrasts greatly with the US where its use was implemented after data on only a few thousand patients. Although, at times, such caution can be praiseworthy, in this case it might also have contributed to these mortality differences.

Important questions still need to be answered, such as the optimal duration and schedule of trastuzumab, and its use with concurrent or sequential chemotherapy. There are cardiotoxicity concerns with the use of trastuzumab, although early indications suggest this occurs when trastuzumab is used in combination with anthracyclines. Nonetheless, while we do not know all the answers to these questions, for women with primary breast cancer, the benefits of this treatment certainly seem to outweigh the risks. All women should be informed of the risk/ benefit ratio, and it is essential that patients are carefully monitored for cardiotoxic effects. If we are intent on improving our breast cancer mortality statistics in the UK, we should now be in the position where this treatment can be offered to patients as readily as it is in other countries.

Supplementary information, in the form of a reference list, is available on the Nature Clinical Practice Oncology website. 